\title{
An Influence-Driven Feedback System For Preference Similarity Network Clustering Based Consensus Group Decision Making Model
}

\author{
Nor Hanimah Kamis ${ }^{\mathrm{a}, \mathrm{b}}$, Francisco Chiclana ${ }^{\mathrm{c}, \mathrm{d}}$, Jeremy Levesley ${ }^{\mathrm{a}}$ \\ ${ }^{a}$ Department of Mathematics, University of Leicester, Leicester, UK \\ ${ }^{b}$ Department of Mathematics, Faculty of Computer and Mathematical Sciences, Universiti Teknologi MARA \\ (UiTM), Shah Alam, Malaysia \\ ${ }^{c}$ Institute of Artificial Intelligence, De Montfort University, Leicester, UK \\ ${ }^{d}$ Andalusian Research Institute on Data Science and Computational Intelligence (DaSCI), University of \\ Granada, Granada, Spain
}

\begin{abstract}
Consensus group decision making (CGDM) allows the integration within this area of study of other advanced frameworks such as Social Network Analysis (SNA), Social Influence Network (SIN), clustering and trust-based concepts, among others. These complementary frameworks help to bridge the gap between their corresponding theories in such a way that important elements are not overlooked and are appropriately taken into consideration. In this paper, a new influence-driven feedback mechanism procedure is introduced for a preference similarity network clustering based consensus reaching process. The proposed influence-driven feedback mechanism aims at identifying the network influencer for the generation of advices. This procedure ensures that valuable recommendations are coming from the expert with most similar preferences with the other experts in the group. This is achieved by adapting, from the SIN theory into the CGDM context, an eigenvector-like measure of centrality for the purpose of: (i) measuring the influence score of experts, and (ii) determining the network influencer. Based on the initial evaluations on a set of alternatives provide by the experts in a group, the proposed influence score measure, which is named the $\sigma$-centrality, is used to define the similarity social influence network (SSIN) matrix. The $\sigma$-centrality is obtained by taking into account both the endogenous (internal network connections) and exogenous (external) factors, which means that SSIN connections as well as the opinion contribution from third parties are permitted in the nomination of the network influencer. The influence-driven feedback mechanism process is designed based on the satisfying of two important conditions to ensure that (1) the revised consensus degree is above the consensus threshold and that (2) the clustering solution is improved.
\end{abstract}

Keywords: Consensus, Preference Similarity, Agglomerative Hierarchical Clustering, Social Influence Network, Centrality, Feedback Mechanism.

\section{Introduction}

Experts in consensus group decision making (CGDM) aim to achieve a common solution with acceptable group agreement. Among the factors that may difficult the required group agreement or consensus level are the experts' background, culture, expertise and motivation. This is because each expert aspires to get his/her opinion to be appropriately considered by the group [1]. New CGDM proposals have been introduced in recent years to deal with this issue,

Email addresses: nk223@le.ac.uk, norhanimah@tmsk.uitm.edu.my (Nor Hanimah Kamis), chiclana@dmu.ac.uk; inv.chiclana@ugr.es (Francisco Chiclana), jl1@leicester.ac.uk (Jeremy Levesley) 
among which it is worth mentioning the following: stochastic optimization cost based CGDM [2], multi-stage fluctuation utility consensus procedure [3], correlation consensus measure [4], hesitant based fuzzy CGDM [5], bipolar consensus approach [6], preferential voting consensus models [7], consensus modeling with cost chance constraint under uncertainty [8], multi-stage fluctuation utility consensus measure [3] and consensus approaches with utility preferences and limited budget [9].

An interesting approach of recent development to deal with the CGDM problem is based on the application of clustering methodologies and derived definitions of useful concepts aiming at presenting homogeneity of experts preferences as a whole: internal and external cohesions, cluster consensus, level consensus and global cluster consensus of the group of experts. Within this approach we can highlight the clustering-based consensus reaching processes proposed by Garcia-Lapresta and Perez-Roman [10, 11, and the partition technique applied by Abel et al. [12] that use experts' opinion similarities and dissimilarities in grouping a set of experts into subgroups to reach a compromise in determining the final group solution. Kamis et al. [13, 14. also advanced the use of preference similarity in deriving a clustering-based CGDM by building an undirected weighted preference similarity network between the group of experts based on the 'structural equivalence' concept and the agglomerative hierarchical clustering algorithm.

Recently, researchers have shown an interest in developing CGDM frameworks to effectively cater for decision making problems with large-scale users and real-time communications, analysing structure of opinion exchange, users interaction pattern and trust relations formation. A systematic review of existing approaches on CGDM within a social network context [15-18] was carried out by Herrera-Viedma et al. [19]. The evolution of CGDM in social networks has led to the development of another relevant area of study, Social Influence Network (SIN). The influence element in SIN plays an important role in CGDM as a key representation of network connection strengths [20], and it has been applied in estimating missing preferences [21], in the evolution of preferences [22] and in the estimation of experts' importance degrees [23, 24].

Most CGDM methods include a feedback mechanism when the group consensus level is not acceptable. This mechanism simulates a discussion medium where experts revisit their evaluations based on the recommendations provided by a 'moderator or leader'. Usually, experts who contribute low to consensus are identified and each of them will get an advice on how to change his/her opinion in order to increase the group consensus level, and eventually to achieve an acceptable group agreement. There exist numerous proposed procedures on implementing a feedback mechanism and advice generation in CGDM. Wu and Chiclana [25] introduced a visual information feedback mechanism by providing graphical representations of experts' current status and simulation of future consensus status after their initial evaluations were replaced with the provided recommendation values. The idea of visualizing consensus state before and after the feedback mechanism was also adopted by Liu et al. [26], where advices were generated by means of the implementation of a trust-based induced feedback recommendation system. Similar feedback approaches and advice generation systems in CGDM can be found in Wu et al. [27] and Gong et al. [28].

This study contributes to consensus generation within a social network framework by introducing a new influence-driven feedback mechanism. The concept of eigenvector-like centrality measure from Social Influence Network (SIN) is proposed to be used and implemented within a preference similarity network clustering based consensus group decision making model. A new centrality influence score measure is developed for the purpose of identifying the network influencer, who will act as the leader in a new Similarity Social Influence Network (SSIN) feedback mechanism. The proposed influence-based concept is also utilized in an IOWA [29] based fuzzy majority dominance resolution process [30] of the CGDM problem to guarantee that the best alternative of consensus is selected by the majority of the experts as a whole.

The rest of the paper is organized as follows: Section 2 introduces the concept of reciprocal 
fuzzy preference relation and its associated intensity preference vector, while Section 3 presents the preference similarity network clustering based consensus reaching process. Section 4 describes the background of social influence theory in decision making context and presents in detail the development of the proposed influence-driven feedback mechanism with its practical implementation steps within a CGDM. An influence-driven resolution process of a CGDM and the general research flow of the proposed model for the influence-based feedback preference similarity network clustering based consensus group decision making problem are provided in Section 5, Conclusions are drawn in Section 6.

\section{Preliminaries on reciprocal fuzzy preference relations}

In solving a consensus decision making problem with a finite set of feasible alternatives, $\mathbb{A}=\left\{A_{1}, A_{2}, \ldots, A_{n}\right\}(n>2)$, a group of experts $E=\left\{e^{1}, e^{2}, \ldots, e^{m}\right\}$ uses their knowledge, experience and expertise to express their opinions with the aim of achieving a final decision with sufficient agreement from every group member. When pairwise comparing alternatives, experts assign to each alternative an intensity of preference degree towards any other alternative [31]. Mathematically, this preference representation is modelled using a reciprocal fuzzy preference relation $(\mathrm{RFPR})$ on $\mathbb{A}$, i.e. a fuzzy binary relation $\mu_{P}: \mathbb{A} \times \mathbb{A} \longrightarrow[0,1]$ that associated each pair of alternatives $\left(A_{i}, A_{j}\right)$ a value $\mu_{P}\left(A_{i}, A_{j}\right)=p_{i j}$ verifying the reciprocity property $p_{i j}+p_{j i}=1(\forall i, j)$ and the following interpretation:

$$
\begin{array}{ll}
p_{i j}=0.5 & \text { if } A_{i} \text { and } A_{j} \text { are equally preferred (indifference) } \\
p_{i j} \in(0.5,1) & \text { if } A_{i} \text { is slightly preferred to } A_{j} \\
p_{i j}=1 & \text { if } A_{i} \text { is absolutely preferred to } A_{j}
\end{array}
$$

An RFPR on a set of alternatives $\mathbb{A}$ can therefore be represented using a matrix of dimension $n \times n, P=\left(p_{i j}\right)$. The set of RFPRs of dimension $n \times n$ will be denoted as $\mathbb{P}_{n \times n}$. Reciprocity property allows the representation of an RFPR in terms of its intensity preference vector (IPV) [4], $V \in \mathbb{R}^{n(n-1) / 2}$ :

$$
V=\left(p_{12}, p_{13}, \ldots, p_{1 n}, p_{23}, \ldots, p_{2 n}, \ldots, p_{(n-1) n}\right)=\left(v_{1}, v_{2}, \ldots, v_{r}, \ldots, v_{n(n-1) / 2}\right) .
$$

The set of IPVs from all experts $E$ on the set of alternatives $\mathbb{A}$ will be denoted by $\mathbb{V}=$ $\left\{V^{1}, V^{2}, \ldots, V^{m}\right\}$.

Example 1. "For the purpose of demonstrating our proposed model, we utilize the existing hypothetical example in [22] where eight (8) experts, $E=\left\{e^{1}, e^{2}, \ldots, e^{8}\right\}$, express their initial evaluations over six (6) alternatives, $\mathbb{A}=\left\{A_{1}, A_{2}, \ldots, A_{6}\right\}$. The RFPR of expert $e^{1}, P^{1}$, and its associated IPV, $V^{1}$, are:

$$
P^{1}=\left[\begin{array}{cccccc}
1 & \mathbf{0 . 2 7} & \mathbf{0 . 6 4} & \mathbf{0 . 8 9} & \mathbf{0 . 7 4} & \mathbf{0 . 8 2} \\
0.73 & 1 & \mathbf{0 . 8 0} & \mathbf{0 . 9 2} & \mathbf{0 . 8 5} & \mathbf{0 . 8 9} \\
0.36 & 0.20 & 1 & \mathbf{0 . 8 5} & \mathbf{0 . 6 2} & \mathbf{0 . 7 4} \\
0.11 & 0.08 & 0.15 & 1 & \mathbf{0 . 2 0} & \mathbf{0 . 2 7} \\
0.26 & 0.15 & 0.38 & 0.80 & 1 & \mathbf{0 . 6 4} \\
0.18 & 0.11 & 0.26 & 0.73 & 0.36 & 1
\end{array}\right]
$$

$V^{1}=(0.27,0.64,0.89,0.74,0.82,0.80,0.92,0.85,0.89,0.85,0.62,0.74,0.20,0.27,0.64)$. 
The IPV of the rest of experts $\left\{V^{2}, V^{3}, \ldots, V^{8}\right\}$ are:

$$
\begin{aligned}
& V^{2}=(0.80,0.85,0.73,0.92,0.89,0.62,0.36,0.85,0.74,0.26,0.80,0.64,0.89,0.82,0.27) ; \\
& V^{3}=(0.69,0.13,0.20,0.36,0.89,0.06,0.10,0.20,0.78,0.63,0.79,0.98,0.69,0.97,0.94) ; \\
& V^{4}=(0.10,0.36,0.69,0.16,0.27,0.83,0.95,0.63,0.76,0.79,0.25,0.39,0.08,0.14,0.65) ; \\
& V^{5}=(0.56,0.15,0.62,0.69,0.93,0.18,0.85,0.60,0.82,0.82,0.93,0.97,0.54,0.82,0.81) ; \\
& V^{6}=(0.17,0.18,0.33,0.45,0.59,0.47,0.61,0.71,0.82,0.60,0.70,0.61,0.62,0.46,0.31) ; \\
& V^{7}=(0.30,0.40,0.45,0.51,0.66,0.53,0.45,0.73,0.68,0.40,0.54,0.66,0.44,0.60,0.37) ; \\
& V^{8}=(0.75,0.75,0.72,0.77,0.82,0.55,0.62,0.59,0.65,0.60,0.46,0.67,0.20,0.44,0.50) . "
\end{aligned}
$$

\section{Preference similarity network clustering based consensus measurement}

In this section, the preference similarity network clustering based consensus decision making procedure used in [14] is referred to, which is described in detail in [13]. Using $\mathbb{V}$, the preference similarity between any pair of experts can be measured and a network structure between the experts in the group $E$ can be created. Formally, a preference similarity measure can be defined as follows:

Definition 1. "Let $\mathbb{V}$ be the set of IPVs of the group of experts $E$ over the set of alternatives $\mathbb{A}$. A preference similarity measure on $E$ is a fuzzy subset of $\mathbb{V} \times \mathbb{V}$ with membership function $S: \mathbb{V} \times \mathbb{V} \rightarrow[0,1]$ verifying both the reflexive $\left[S\left(V^{a}, V^{a}\right)=1\right]$ and the symmetric $\left[S\left(V^{a}, V^{b}\right)=S\left(V^{b}, V^{a}\right)\right]$ properties."

As per Definition 1, an indirect preference similarity network, $\mathbb{N}=\langle E, T, \mathbb{S}\rangle$, between the experts (nodes) in $E$ can be created with the preference similarity degree between experts, $\mathbb{S}=\left(S_{1}, S_{2}, \ldots, S_{m(m-1) / 2}\right)$, as the set of weights attached to the set of ties between nodes, $T=\left(t_{12}, t_{13}, \ldots, t_{1 n}, t_{23}, \ldots, t_{2 m}, \ldots, t_{(m-1) m}\right)$, to express the strength of the similarity relation between the network nodes (experts' preferences). In Social Network Analysis (SNA), two nodes are structurally equivalent if they have the same neighbors and similar characteristics in their social environments [32]. The cosine similarity function can be used to achieve the structural equivalence preference similarity network:

$$
S^{a b}=S\left(V^{a}, V^{b}\right)=\frac{\sum_{i=1}^{n(n-1) / 2}\left(v_{i}^{a} \cdot v_{i}^{b}\right)}{\sqrt{\sum_{i=1}^{n(n-1) / 2}\left(v_{i}^{a}\right)^{2}} \cdot \sqrt{\sum_{i=1}^{n(n-1) / 2}\left(v_{i}^{b}\right)^{2}}} .
$$

For the purpose of categorizing structurally equivalence experts in the undirected weighted preference similarity network, the agglomerative hierarchical clustering approach is applied as per Algorithm 1 below. 


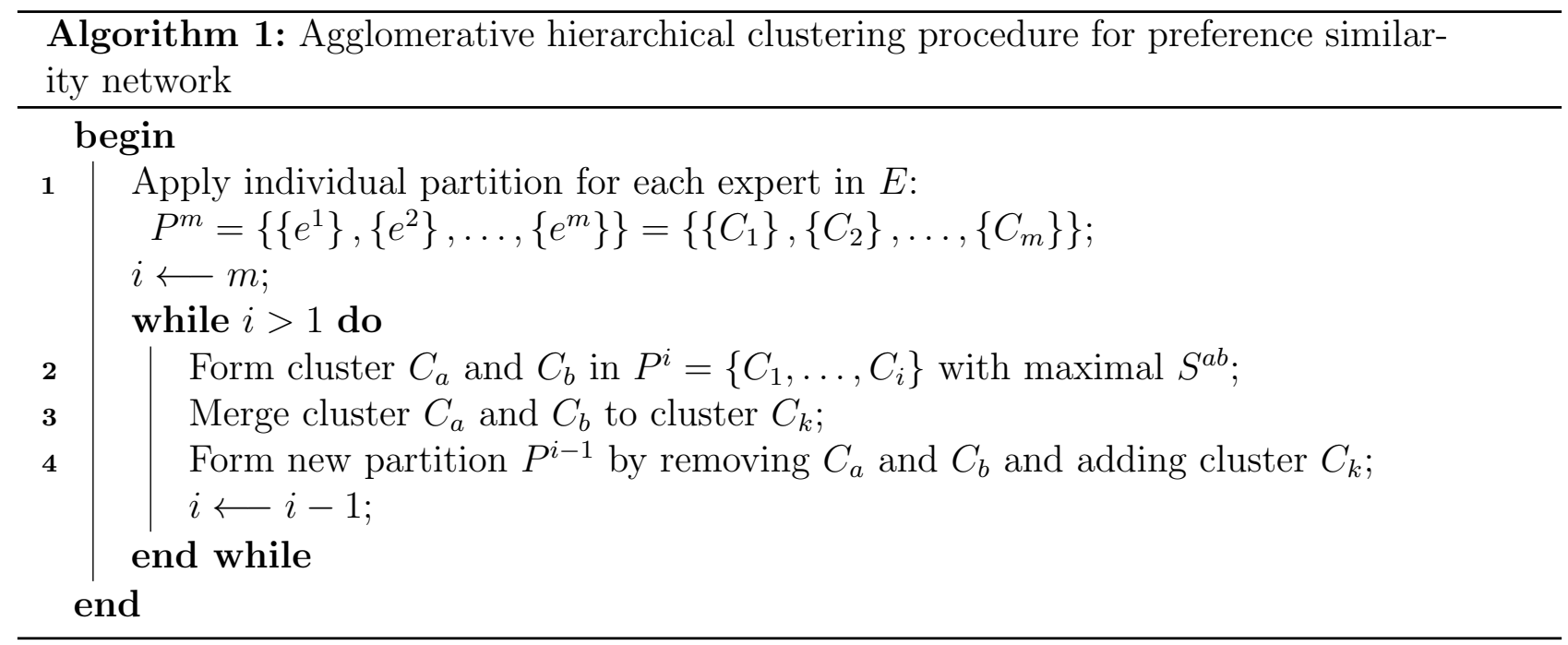

A dendogram is a convenient visualisation of the hierarchical clustering generated by Algorithm 1. Using horizontally cuts at particular $\alpha_{l}$-levels $(l=2, \ldots, m-1)$ in a dendogram, a partition of the experts into a number of clusters $\left(C_{l}\right)$, with respect to the cosine preference similarity matrix $S$, is obtained (see Example 2 ending this section).

This implementation of the structural equivalence concept means that experts in the same cluster have strong connections between them and, therefore, they are expected to reach higher cohesiveness among them than with outsider experts. Thus, as described below, measurements of experts' cluster homogeneity according to the internal and external cohesions can be computed and combined to derive a cluster consensus measure.

Let $L=\left\{\alpha_{l} ; l=2, \ldots, m-1\right\}$ be the set of all different $\alpha_{l}$-levels and $C_{l}=\left\{C_{l k} ; k=1, \ldots, l\right\}$ the set of clusters at the $\alpha_{l}$-level, with cardinality $\sharp C_{l k}$.

- The $\alpha_{l}$-level cluster internal cohesion degree of cluster $C_{l k}$ :

$$
\delta_{\text {int }}\left(C_{l k}\right)=\frac{\sum_{i \in C_{l k}} \sum_{j \in C_{l k}} S^{i j}}{\left(\sharp C_{l k}\right)^{2}}
$$

- The $\alpha_{l}$-level cluster external cohesion degree of cluster $C_{l k}$ :

$$
\delta_{e x t}\left(C_{l k}\right)=\frac{\sum_{i \in C_{l k}} \sum_{j \notin C_{l k}} S^{i j}}{\sharp C_{l k}\left(n-\sharp C_{l k}\right)}
$$

- The $\alpha_{l}$-level cluster consensus degree of cluster $C_{l k}$ :

$$
\delta_{C C}\left(C_{l k}\right)=\frac{\sharp C_{l k} \cdot \delta_{i n t}\left(C_{l k}\right)}{n}+\frac{\left(n-\sharp C_{l k}\right) \cdot \delta_{e x t}\left(C_{l k}\right)}{n}
$$

- The $\alpha_{l}$-level average cluster consensus degree of the group of experts $E$ measures the agreement index between experts of all clusters at that $\alpha_{l}$-level:

$$
\delta_{L C}(l)=\frac{\sum_{k=1}^{l} \delta_{C C}\left(C_{l k}\right)}{l} .
$$


- The global cluster consensus degree of the group of experts $E$ is $\delta_{L C}(\hat{l})$ with $\alpha_{\hat{l}}$-level having the maximum of all $\alpha_{l}$-level average cluster consensus degrees, which is referred to as the optimal consensus agglomerative hierarchical clustering level.

Let the threshold value of sufficient group consensus be set at the value $\varphi$. The preference similarity network clustering based consensus reaching procedure is summarised in Algorithm 2 below.

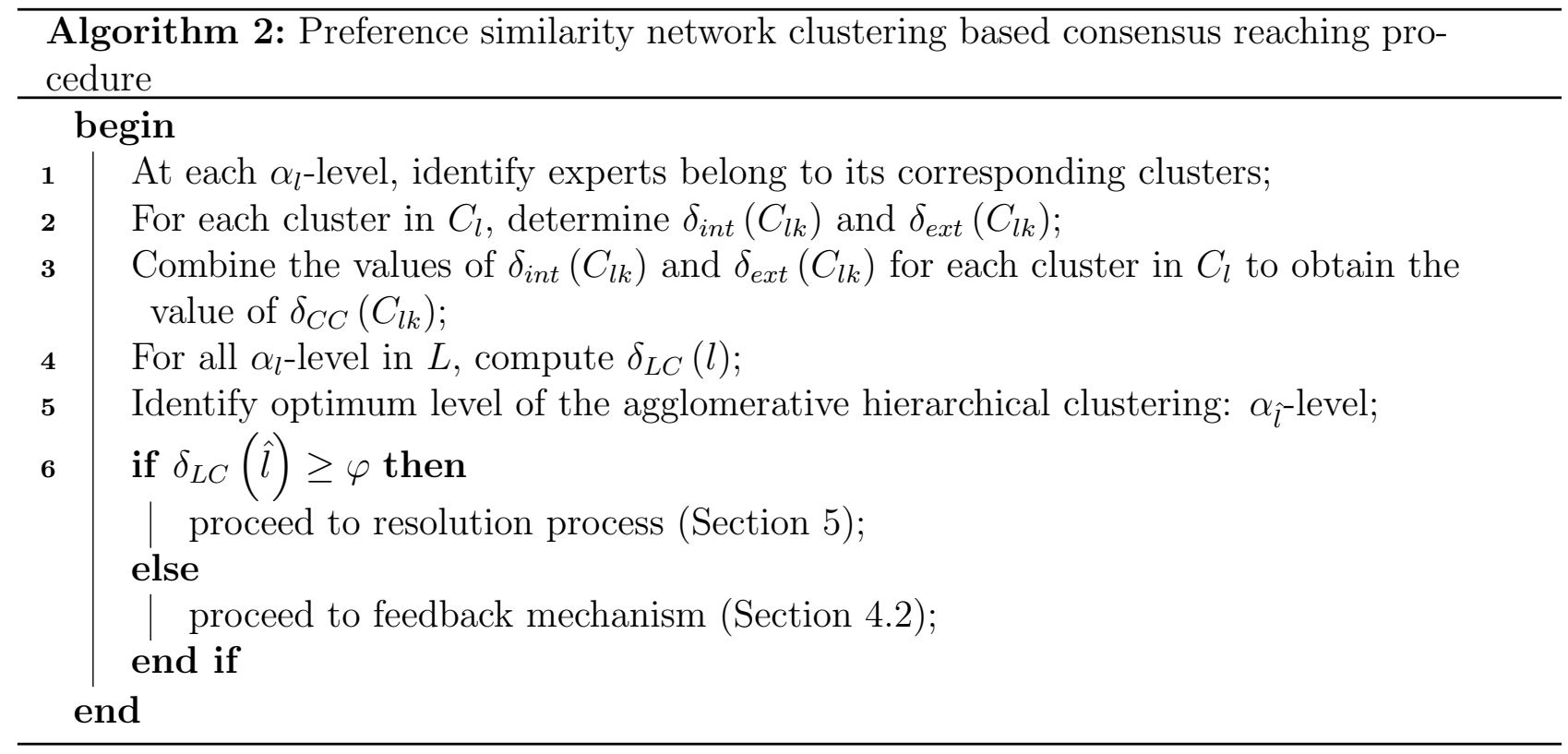

Example 2 (Continuation of Example 1). "Applying (3), the following cosine preference similarity matrix is obtained:

$$
\mathbb{S}=\left[\begin{array}{cccccccc}
1 & 0.868 & 0.742 & 0.937 & 0.906 & 0.926 & 0.945 & 0.957 \\
0.868 & 1 & 0.817 & 0.704 & 0.876 & 0.896 & 0.954 & 0.932 \\
0.742 & 0.817 & 1 & 0.609 & 0.925 & 0.840 & 0.854 & 0.803 \\
0.937 & 0.704 & 0.609 & 1 & 0.797 & 0.844 & 0.834 & 0.840 \\
0.906 & 0.876 & 0.925 & 0.797 & 1 & 0.944 & 0.942 & 0.912 \\
0.926 & 0.896 & 0.840 & 0.844 & 0.944 & 1 & 0.970 & 0.883 \\
0.945 & 0.954 & 0.854 & 0.834 & 0.942 & 0.970 & 1 & 0.945 \\
0.957 & 0.932 & 0.803 & 0.840 & 0.912 & 0.883 & 0.945 & 1
\end{array}\right]
$$

Figure 1 shows the complete graph representation of the undirected weighted preference similarity network between the set of experts, with only a few link weights showed for simplicity reason. The experts' cluster pattern according to the dendogram (Figure 2)) is generated by Algorithm 1 at the optimal $\alpha_{l}$-level identified by Algorithm 2 as detailed in Table 1. The optimum global consensus of 0.883 is unique and achieved at the $\alpha_{7}$-level. If the consensus threshold were above 0.883 (say $\varphi=0.945$ ), then group consensus would be not sufficient and a second round of the consensus process would be required, which is to be preceded by the activation of a feedback process to increase the group consensus. It is noticed that the optimum cluster level results in only 2 experts being grouped in a cluster while the rest are on their own cluster, respectively." 


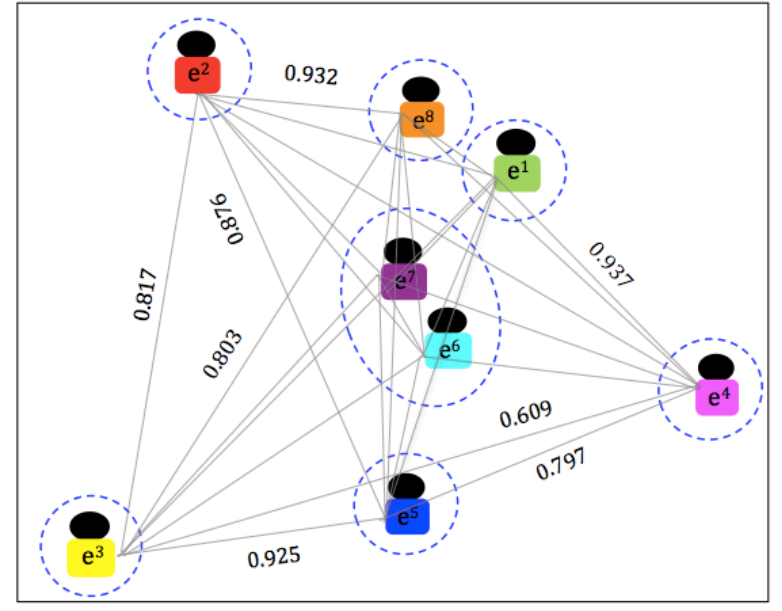

Figure 1: The undirected weighted preference similarity network with experts clustering pattern

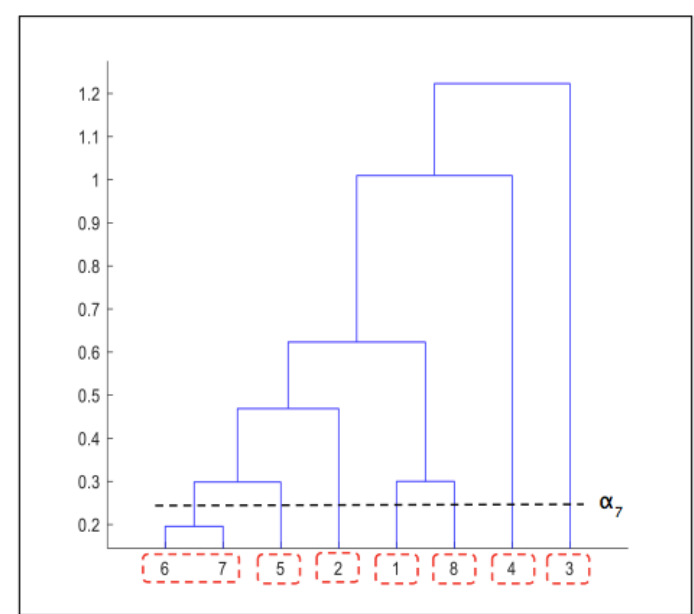

Figure 2: Dendogram generated by Algorithm 1 with the optimal $\alpha_{l}$-level from Algorithm 2

Table 1: Cluster internal and external cohesions degrees, cluster consensus and average cluster consensus degrees

\begin{tabular}{|c|c|c|c|c|c|c|}
\hline$\alpha$ & $C$ & $E$ & $\delta_{\text {int }}$ & $\delta_{e x t}$ & $\delta_{C C}$ & $\delta_{L C}$ \\
\hline \multirow[t]{2}{*}{2} & 1 & $e^{1}, e^{2}, e^{4}, e^{5}, e^{6}, e^{7}, e^{8}$ & 0.911 & 0.799 & 0.897 & 0.860 \\
\hline & 2 & $e^{3}$ & 1 & 0.799 & 0.824 & \\
\hline \multirow[t]{3}{*}{3} & 1 & $e^{1}, e^{2}, e^{5}, e^{6}, e^{7}, e^{8}$ & 0.937 & 0.828 & 0.909 & 0.851 \\
\hline & 2 & $e^{4}$ & 1 & 0.795 & 0.821 & \\
\hline & 3 & $e^{3}$ & 1 & 0.799 & 0.824 & \\
\hline \multirow[t]{4}{*}{4} & 1 & $e^{2}, e^{5}, e^{6}, e^{7}$ & 0.948 & 0.871 & 0.909 & 0.866 \\
\hline & 2 & $e^{1}, e^{8}$ & 0.979 & 0.887 & 0.910 & \\
\hline & 3 & $e^{4}$ & 1 & 0.795 & 0.821 & \\
\hline & 4 & $e^{3}$ & 1 & 0.799 & 0.824 & \\
\hline \multirow[t]{5}{*}{5} & 1 & $e^{5}, e^{6}, e^{7}$ & 0.968 & 0.889 & 0.919 & 0.871 \\
\hline & 2 & $e^{2}$ & 1 & 0.864 & 0.881 & \\
\hline & 3 & $e^{1}, e^{8}$ & 0.979 & 0.887 & 0.910 & \\
\hline & 4 & $e^{4}$ & 1 & 0.795 & 0.821 & \\
\hline & 5 & $e^{3}$ & 1 & 0.799 & 0.824 & \\
\hline \multirow[t]{6}{*}{6} & 1 & $e^{5}, e^{6}, e^{7}$ & 0.968 & 0.889 & 0.919 & 0.877 \\
\hline & 2 & $e^{2}$ & 1 & 0.864 & 0.881 & \\
\hline & 3 & $e^{1}$ & 1 & 0.898 & 0.910 & \\
\hline & 4 & $e^{8}$ & 1 & 0.896 & 0.909 & \\
\hline & 5 & $e^{4}$ & 1 & 0.795 & 0.821 & \\
\hline & 6 & $e^{3}$ & 1 & 0.799 & 0.824 & \\
\hline \multirow[t]{7}{*}{7} & 1 & $e^{6}, e^{7}$ & 0.985 & 0.901 & 0.922 & 0.883 \\
\hline & 2 & $e^{5}$ & 1 & 0.900 & 0.913 & \\
\hline & 3 & $e^{2}$ & 1 & 0.864 & 0.881 & \\
\hline & 4 & $e^{1}$ & 1 & 0.898 & 0.910 & \\
\hline & 5 & $e^{8}$ & 1 & 0.896 & 0.909 & \\
\hline & 6 & $e^{4}$ & 1 & 0.795 & 0.821 & \\
\hline & 7 & $e^{3}$ & 1 & 0.799 & 0.824 & \\
\hline
\end{tabular}




\section{Social influence theory in the CGDM context}

We begin this section by reviewing related social influence studies implemented in CGDM frameworks. Our proposed influence-guided feedback mechanism methodology is presented and the second consensus round is then elaborated.

\subsection{Review on existing influence methodologies in CGDM}

Social influence network (SIN) has been developed continuously since the 1950s by French [33], Harary [34, DeGroot [35] and Friedkin and Johnsen [36]; it is one of the important fields directly linked to group decision making and SNA.

Leading to this correlated direction, recent studies on CGDM with incorporation of social influence theory, derived from social networks, have been introduced. Brunelli et al. [16] addressed consensus evaluation by considering experts' influence strengths in a social network via an eigenvector centrality measure with a fuzzy $m$-ary adjacency relation approach. In [17, a leadership-based consensus reaching procedure was developed where opinion managers are allowed to give advices and influence the opinion formation from a social network towards achieving consensus. Another study worth mentioning was carried out by Liang et al. [20], where the authors modelled the social influence in terms of experts' tie strengths in a social network, with the number of common members' connections were combined with the number of their direct interactions.

The influence factor is necessary to be implemented in CGDM frameworks in order to ensure the acceptance by the experts of the recommendation advices produced by a feedback mechanism algorithm to increase consensus. By doing so, advices can be generated from the most influential person in the network, which implicitly brings attached a trust statement with it. It has been argued that a feedback mechanism guided by trust relationships is more realistic than the traditional feedback mechanism because the generated advices are more persuasive and acceptable by a group of experts [18, 24, 26, 37].

Perez et al. 22, and Capuano et al. 21] modelled influence processes in GDM by utilising the recursive approach presented in Friedkin and Johnsen [38]. Let $E$ be a set of experts, $W=\left(w_{i j}\right)_{m \times m}$ a fuzzy adjacency matrix representing experts' relative importance on others' preferences including themselves, and $y^{1}$ the initial experts' preferences. After $t$ iterations, the influenced generated preferences will be

$$
y^{t}=A W y^{(t-1)}+(I-A) y^{1},
$$

where $A=\operatorname{diag}\left(a_{11}, a_{22}, \ldots, a_{m m}\right)$ is the susceptibility of experts to interpersonal influences and $I$ is the $m \times m$ identity matrix. Significantly, the above expression allows to estimate the evolution of experts' preferences iteratively until the process reaches an equilibrium, i.e. $y^{\infty}=\lim _{t \rightarrow \infty} y^{t}$ exists and it is expressed as follows:

$$
y^{\infty}=(I-A W)^{-1}(I-A) y^{1} .
$$

Capuano et al.'s work [21] provided greater flexibility compared to Perez et al.'s [22]. Capuano's proposed model proved that the complication of defining a numerical level expressing the susceptibility of experts towards influence can be avoided with the estimation of missing preferences been successfully operated and the convergence of experts' preferences achieved.

An alternative influence measure, known as alpha-centrality, was introduced by Bonancich and Lloyd [39]. The alpha-centrality, denoted here by $x$, is an eigenvector-like measure that determines expert's status in a network by considering their influence pattern; it is expressed as follows:

$$
x=\left(I-\Upsilon \boldsymbol{A}^{T}\right)^{-1} \boldsymbol{e}
$$


where $\boldsymbol{A}=\left(a^{i j}\right)$ is an adjacency matrix that represents the group's influence pattern, i.e. $a^{i j}$ represents the degree of influence of expert $i$ by expert $j$; $\Upsilon$ is a scalar describing the relative importance degree of endogenous (internal) versus exogenous (external) effects of experts in a group. Bonacich and Lloyd suggested that $\Upsilon$ should approach $1 / \lambda_{1}$ from below in order to achieve convergence of the solution, with $\lambda_{1}$ being the largest eigenvalue of $\boldsymbol{A}$. Notice that the endogenous factor emerges from connections in the network itself while the exogenous factor, $\boldsymbol{e}$, is external to the network of experts, such as from third party involvements, and that it can affect or change the experts' status. For example, the selection of the best employer will involve peer reviews, which can be presented as a relationship network in a workplace and this criteria is considered as an endogenous effect. In some situation, the selection process involves evaluation from company's top management, which can be seen an exogenous factor in the nomination of the best employer.

\subsection{A new social influence-based feedback mechanism}

Social influence models focus on the development of interpersonal influence processes, where experts are allowed to manage the conflicting influential preferences by revising and inducing it to behave in a similar way to others for the purpose of achieving group consensus. There exist two main aspects to be explored in social influence theory [38]: (1) how experts change their preferences and influence the other experts; and (2) how to develop structure of social influence models with better configuration and strengthen the interpersonal influence processes.

In a group decision making context, social influence is clarified by changes of attitudes, thoughts, feelings, characters or behaviors of expert(s), if there exists interaction from another expert in the group [20]. This situation allows modification of experts' preferences due to the social influence factor during interactions, discussions or opinion exchange in a network. Experts with strong knowledge, experience, background, trustworthy and motivation are able to act as 'leaders' in the network, in such a way that they can influence the others to move in the similar direction towards group consensus. This can be done by implementing a specific procedure in CGDM, known as 'feedback mechanism module'. Practically, a feedback mechanism must have flexible acceptance of the recommendation advices according to the affordability of the adjustment cost and experts guidance in order to choose the optimal feedback parameter for the purpose of balancing the individual adjustment cost with the group consensus [40].

The proposed novel influence-based feedback mechanism comprises three main phases: (1) identification of expert(s) with low consensus contribution; (2) identification of a network influencer; and (3) generation of advice. It is implemented in two different situations: (a) without exogenous effect; and (b) with exogenous effect. Explanations on this feedback process are presented below.

\subsubsection{Identification of expert(s) with low consensus contribution}

The global cluster consensus degree, $\delta_{L C}(\hat{l})$, is the average cluster consensus degree of the experts at the optimal consensus agglomerative hierarchical clustering $\alpha_{\hat{l}}$-level. At this level, those experts in a cluster $C_{\hat{l} k}$ with $\alpha_{\hat{l}}$-level cluster consensus below the global cluster consensus degree will be identified as contributing low to consensus (below the average). This is formulated as follows:

$$
\begin{gathered}
e_{\text {low }}=\left\{e^{o} \in E \mid e^{o} \in C_{\hat{l} k} \wedge C_{\hat{l} k} \in C_{\text {low }}\right\}, \\
C_{\text {low }}=\left\{C_{\hat{l} k} \mid \delta_{C C}\left(C_{\hat{l} k}\right)<\delta_{L C}(\hat{l}) \wedge k=1, \ldots, \hat{l}\right\} .
\end{gathered}
$$

Experts in $e_{\text {low }}$ will be adviced on how to change their opinions in order to increase the consensus level. This is proposed to be done by identifying the network influencer using influence-driven concepts from SIN theory. 


\subsubsection{Identification of the network influencer}

For the purpose of ensuring recommendations or advices perform well in decision making process, we adapt a 'memory-based collaborative filtering' idea [41] into SIN, where historical/initial data of a group of users are used to provide 'valuable recommendations coming from someone who has shared similar history with other people in a group' [42. According to this concept, we utilize the experts' initial evaluations (Section 2) as historical data to obtain the experts' preference similarity matrix, $\mathbb{S}$, as a criterion to be composed in our proposed SIN, which is constructed by a digraph that links the set of experts $E$ (nodes) with edge connecting expert $e^{i}$ and $e^{j},\left(e^{i}, e^{j}\right)$, with the influence strength weight of the $j^{\text {th }}$ expert over the $i^{\text {th }}$ expert.

In our case, the SIN comprises a set of experts, $E=\left\{e^{1}, e^{2}, \ldots, e^{8}\right\}$, and a row normalized preference similarity matrix, $\mathbb{S}_{\eta}=\left(S_{\eta}^{i j}\right)_{n \times n}$, where $S_{\eta}^{i j}$ is the proportion of overall group influence on $i$ that comes from $j$. Notice that $\mathbb{S}_{\eta}$ is obtained by taking a row normalization step on the preference similarity matrix (Section 3 ), $\mathbb{S}$, so that the following property $\sum_{j=1}^{n} S_{\eta}^{i j}=1$ for all $i \in(1, \ldots, n)[21]$ is verified. This property ensures the influence of each expert towards all of his/her peers is 1 in total. Formally, we name our SIN as Similarity Social Influence Network $(S S I N)$, which is visualised for a simple case of 3 expert nodes in Fig. 3 and defined below:

Definition 2. "A Similarity Social Influence Network (SSIN) is an ordered 3-tuple, G= $\left\langle E, L, \mathbb{S}_{\eta}\right\rangle$, comprising a set of nodes $(E)$, a set of edges $(L)$ or ordered pairs of experts in $E$, and a set of row normalized preference similarity weights $\left(\mathbb{S}_{\eta}\right)$ attached to L."

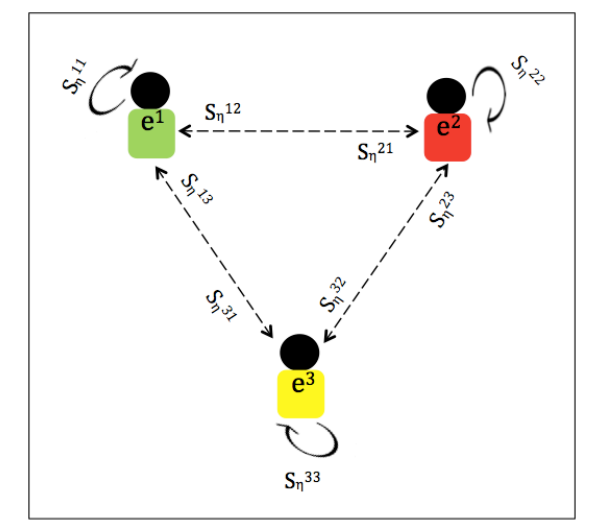

Figure 3: The general representation of SSIN consisting 3-expert nodes

In this study, we make use of the previously described influence measure by Bonacich and Lloyd [39]. It is adapted to our preference similarity network clustering based consensus group decision making model in order to identify the most influential expert of the network. The identified influencer will act as the 'leader' in designing feedback rules with the aim to increase the group consensus level when this is below a satisfactory threshold value. We name our proposed influence measure the $\sigma$-centrality and defines it formally below:

Definition 3. "Let $\mathbb{S}_{\eta}$ be a set of row normalized preference similarity weights in SSIN G, $\sigma$ the relative importance of endogenous (network connections) versus exogenous (external) effects, and $Z=(z)_{m \times 1}$ a set of individual expert exogenous effect values. Then, the influence score or $\sigma$-centrality of experts $E, Y=\left(y^{1}, \ldots, y^{m}\right)$, is:

$$
Y=\left(I-\sigma \mathbb{S}_{\eta}^{T}\right)^{-1} Z
$$

In the absence of exogenous effect, $Z$ is set as the unity vector, i.e. the vector with all components equal to $1 . "$ 
The combination of these two factors, endogenous and exogenous, produce stability in generating experts' influence scores because it is dependent on internal contributions to consensus from the generated preference similarity network and third-party importance evaluations over experts when available. Indeed, the value of $Y$ represents the influence score of each expert, in such a way that both endogenous (internal) and exogenous (external) factors are considered.

Experts who fall in clusters having higher cluster consensus degrees than or equal to the global cluster consensus degree of the group can be classified as belonging to the group of most influential experts in the network. Let $C_{\hat{l} k}^{*}$ be set of clusters at the optimum clustering $\alpha_{\hat{l}}$-level with cluster consensus indexes, $\delta_{C C}(k)$, above the global cluster consensus degree of the group of experts, $\delta_{L C}(\hat{l})$, and $e_{\hat{l} k}^{y^{*}}$ be the experts belonging to the clusters in $C_{\hat{l} k}^{*}$. The identification of possible network influencers can be formally written as:

$$
\begin{gathered}
e_{\hat{l} k}^{y^{*}}=\left\{e^{y} \mid e^{y} \in C_{\hat{l} k} \wedge C_{\hat{l} k} \in C_{\hat{l} k}^{*}\right\}, \\
C_{\hat{l} k}^{*}=\left\{C_{\hat{l} k} \mid \delta_{C C}(k) \geq \delta_{L C}(\hat{l}) \wedge k=1, \ldots, \hat{l}\right\}
\end{gathered}
$$

Thus, the network influencer, $e^{*}$, is the expert with highest influence score among those in $e_{\hat{l} k}^{y^{*}}$ :

$$
e^{*}=\max _{e^{y} \in C_{i k}^{*}} Y\left(e^{y}\right) .
$$

The identified network influencer, $e^{*}$, will act as the advisor for the experts who contribute low to consensus on how to change their opinions with the aim to increase and, subsequently, to reach the group consensus threshold level. This is elaborated in the following section.

\subsubsection{Generation of advice}

As mentioned in Section 4.2.1, for the purpose of increasing the consensus level of the group, an expert $e^{o}$ in $e_{\text {low }}$ is provided (feedback) the following new intensity preference vector $\widetilde{V}^{o}$ :

$$
\widetilde{V}^{o}=(1-\beta) \cdot V^{o}+\beta \cdot V^{*}
$$

where $V^{o}$ is the current IPV of $e^{o}, V^{*}$ is the IPV of the network influencer, $e^{*}$, and $\beta \in[0,1]$ is a control parameter that can be used to adjust the extent of the change of preferences feedback to the experts in $e_{l o w}$.

This section concludes with the below algorithmic representation of the proposed influencebased feedback mechanism procedure.

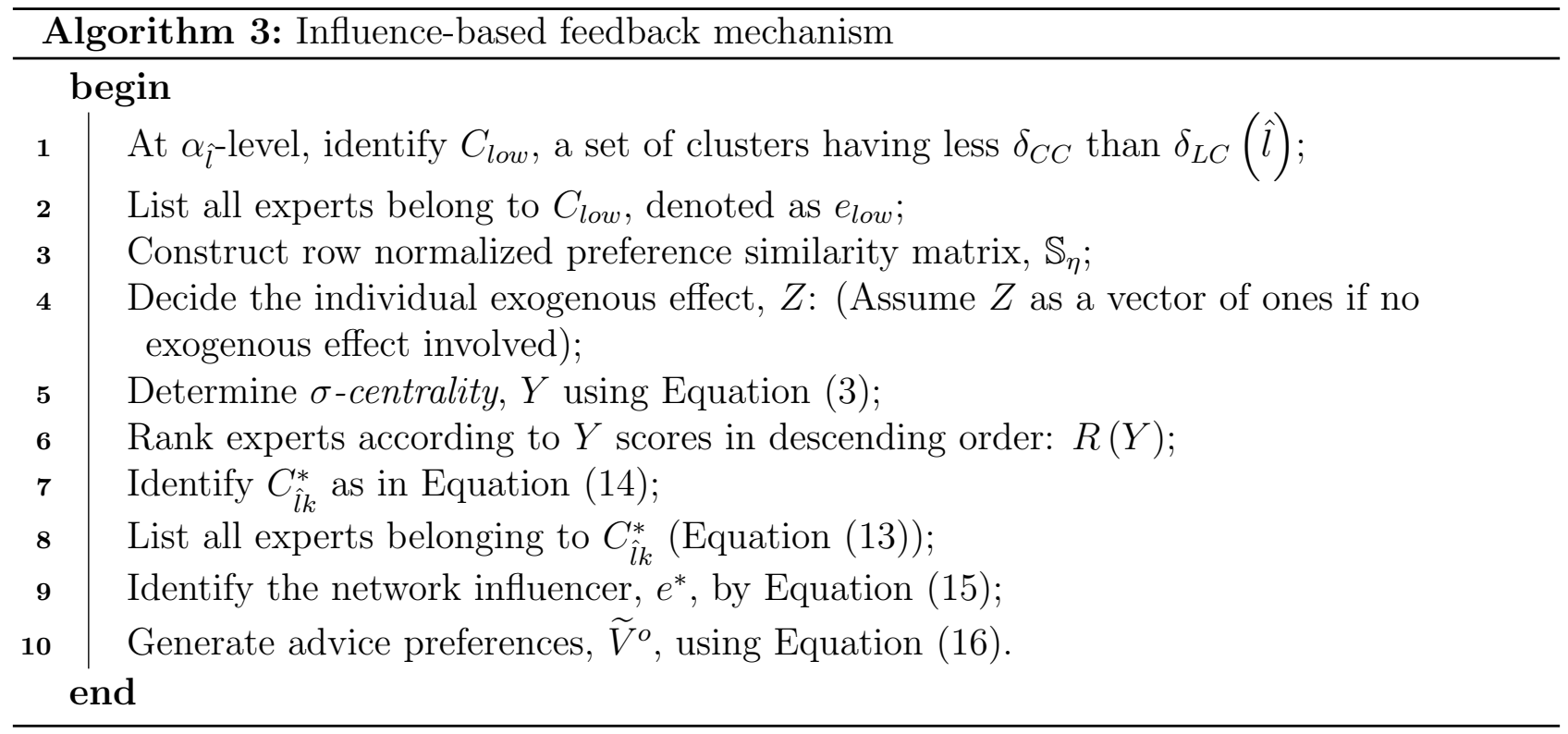




\subsection{Consensus reaching procedure with optimal parameter of control}

If $\beta=0$ no changes are recommended and the original preferences of the expert remain unchanged. If $\beta=1$ then the expert's preferences are completely substituted by those of the network influencer. For the influence-based feedback mechanism to be effective, a control parameter $\beta \in[0,1]$ should be selected to guarantee that the following two conditions are verified:

- the global cluster consensus degree of the group of experts of the second consensus round, $\delta_{L C}^{2}(\hat{l})$ must be greater than or equal the consensus threshold, $\varphi$ and;

- the optimal agglomerative hierarchical clustering level in second round of consensus process, $\alpha_{\hat{l}}^{2}$ must be less than the optimal agglomerative hierarchical clustering level of first round, $\alpha_{\hat{l}}$.

The advice generation with $\beta=0$ produces the first round of consensus solution, and therefore it is not implemented if group consensus is to be achieved. For simplicity, we use discrete values of $\beta$ from the set $\{0.1, \ldots, 0.9,1\}$. The first condition above states that sufficient consensus level will be achieved, while the second one is purposely introduced to achieve a better clustered solution (lower number of clusters) after the implementation of the feedback process. Without imposing restrictions to the parameter of control, the above two conditions will be achieved at some extent because when the feedback advices are implemented, the experts will be more similar because the preferences will be closer the network influencer's preferences, which also will have a positive effect on the cohesiveness within clusters. These two conditions are formally presented in the following definition.

Definition 4. "The revised global cluster consensus degree, $\delta^{2}(\hat{l})$, of the group of experts, E, for the second round of the consensus reaching process satisfies: $\left(\delta_{L C}^{2}(\hat{l}) \geq \varphi\right) \wedge\left(\alpha_{\hat{l}}^{2}<\alpha_{\hat{l}}\right)$."

The algorithm to find the optimal parameter of control, within the discrete set of values $\{0.1, \ldots, 0.9,1\}$, with respect to Definition 4 is presented below:

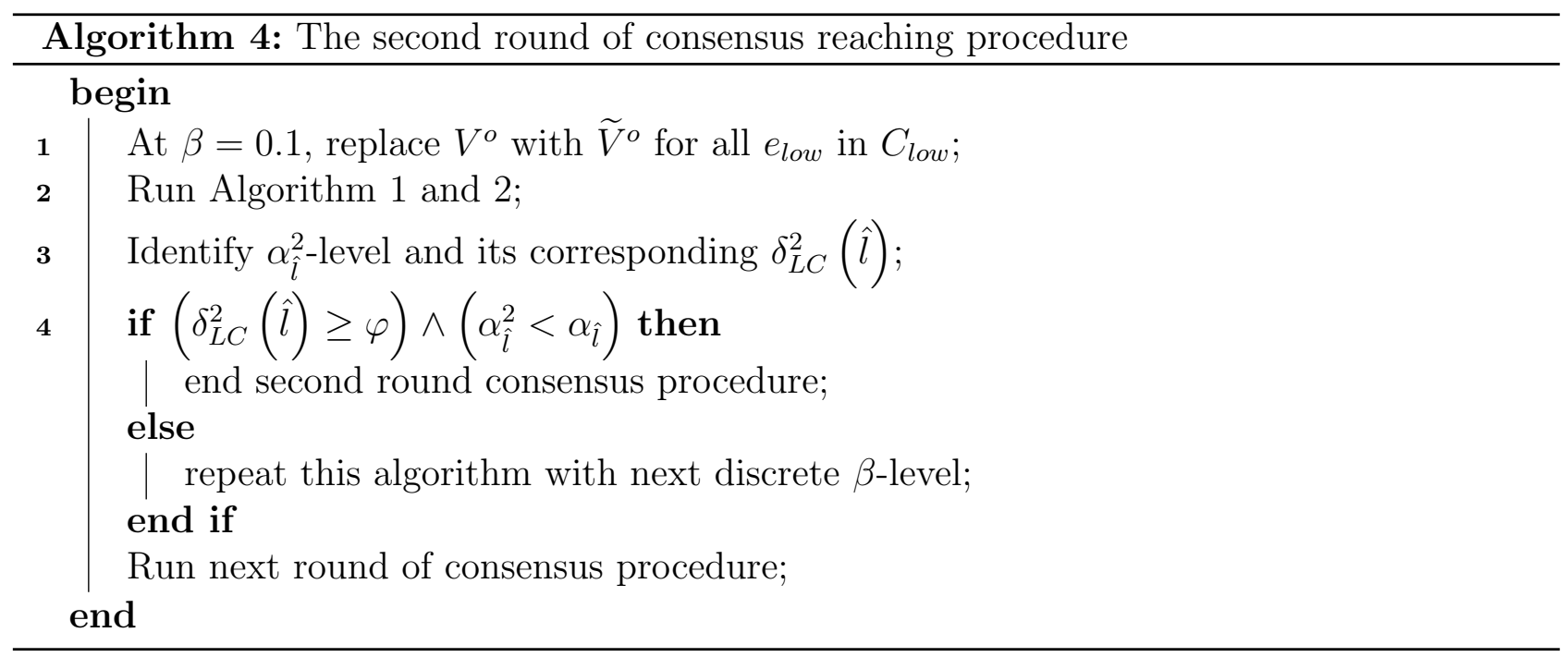

Example 3 (Continuation of Example 2). "By referring to Table 1, the global consensus degree of the group of experts is 0.883 , which is lower than the pre-determined consensus threshold (0.945). It means that the consensus level is insufficient, thus the feedback mechanism is activated. In phase 1 of the proposed influence-based feedback mechanism (Algorithm 3, Step 1 and 2) the experts with low consensus contribution are identified: 
- Clusters having less cluster consensus degrees than 0.883 are $C_{3}, C_{6}$ and $C_{7}$.

- Thus, $e_{\text {low }}=\left\{e^{2}, e^{3}, e^{4}\right\}$.

In phase 2 of the proposed influence-based feedback mechanism (Algorithm 3, Step 3 until 9), the network influencer is identified by computing the influence score or $\sigma$-centrality associated to each expert.

- The row normalized preference similarity matrix, $\mathbb{S}_{\eta}$, is computed and its corresponding SSIN is presented in Figure 4 with only some of the row normalized preference similarity weights displayed for simplicity reason.

$$
\mathbb{S}_{\eta}=\left[\begin{array}{llllllll}
0.137 & 0.119 & 0.102 & 0.129 & 0.124 & 0.127 & 0.130 & 0.131 \\
0.123 & 0.142 & 0.116 & 0.100 & 0.124 & 0.127 & 0.135 & 0.132 \\
0.113 & 0.124 & 0.152 & 0.092 & 0.140 & 0.127 & 0.130 & 0.122 \\
0.143 & 0.107 & 0.093 & 0.152 & 0.121 & 0.128 & 0.127 & 0.128 \\
0.124 & 0.120 & 0.127 & 0.109 & 0.137 & 0.129 & 0.129 & 0.125 \\
0.127 & 0.123 & 0.115 & 0.116 & 0.129 & 0.137 & 0.133 & 0.121 \\
0.127 & 0.128 & 0.115 & 0.112 & 0.127 & 0.130 & 0.134 & 0.127 \\
0.132 & 0.128 & 0.110 & 0.116 & 0.125 & 0.121 & 0.130 & 0.138
\end{array}\right]
$$

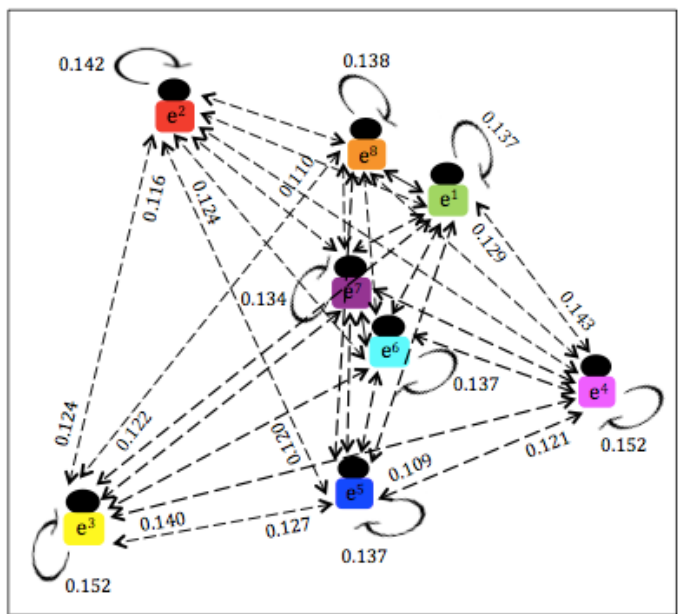

Figure 4: The generated similarity social influence network (SSIN)

- From $\mathbb{S}_{\eta}$, the $\sigma$ value is determined. As mentioned in Section 4.1, the convergence of the solution is assured if $\sigma$ is selected subject to the constraint $\sigma<\frac{1}{\lambda_{1}}$, where $\lambda_{1}$ is the unique largest eigenvalue of $\mathbb{S}_{\eta}$. The eigenvalues of $\mathbb{S}_{\eta}$ are

$$
\{0.998,0.0726,0.0313,0.0158,0.0055,0.0021,0.0007,0.0013\}
$$

and $\lambda_{1}=0.998$. The value $\sigma=0.9$ is selected to fulfil the above boundary constraint.

- Whether exogenous (external) effect plays a role in the SSIN is to be established. The following two cases (refer to Table 2) are discussed:

- Without exogenous effect case with $Z$ set as the unit vector. In this case it is

$$
Y=[0.128,0.124,0.117,0.117,0.128,0.128,0.130,0.128] .
$$

The network influencer would be $e^{7}$. 
- Exogenous effect case with following vector $Z=[1,0.7,0.4,0.8,0.9,0.3,0.5,0.1]$. In this case it is

$$
Y=[0.137,0.126,0.113,0.121,0.135,0.122,0.129,0.117] .
$$

The network influencer would be $e^{1}$.

Table 2 provides a complete comparative view of the results obtained after the feedback mechanism is implemented with and without exogenous effects, respectively.

Table 2: Comparative results of feedback mechanism with and without exogenous effects in $\sigma$-centrality measure

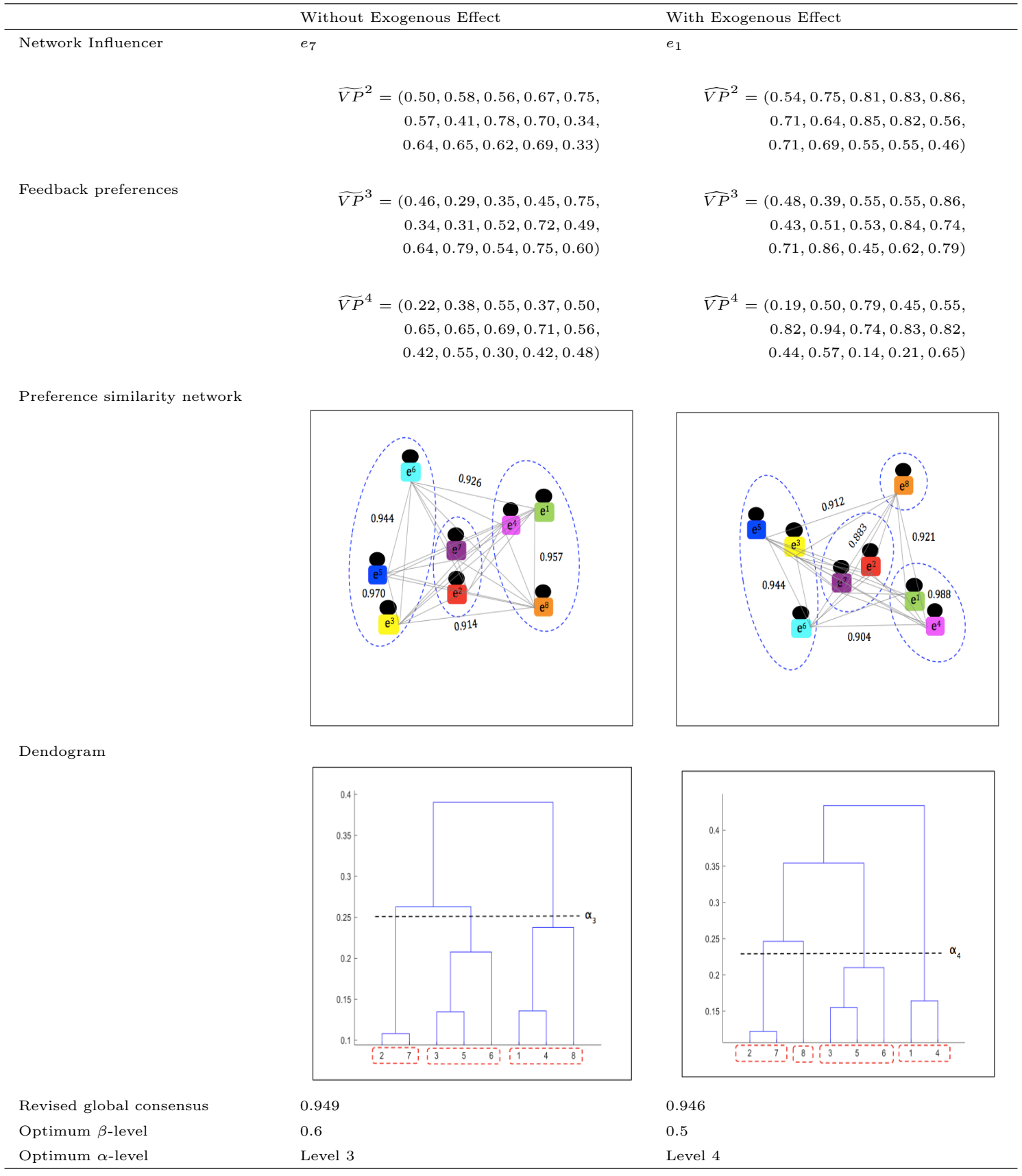

The feedback preference values in the case of exogenous effect are in general greater than in the case without exogenous effect. Obviously, the experts preference similarity degrees increase after the feedback mechanism, which is reflected in the higher revised global cluster consensus of the group of experts. In fact, the consensus threshold set in Example 2. $\varphi=0.945$, is reached in both cases after the feedback process (in the second consensus round): 0.949 with an optimum 
parameter of control $\beta=0.6$ for the case without exogenous effect; and 0.946 with optimum parameter of control $\beta=0.5$ for the case with exogenous effect.

Table 3 provides the computations for the optimal global consensus degrees and their corresponding optimal cluster $\alpha$-levels at each $\beta$-level for the second round of consensus reaching process for both with and without exogenous effects.

Table 3: Optimal global consensus degrees and their corresponding optimal cluster solutions $(\alpha$-levels $)$ at each $\beta$-level for the second round of consensus reaching process, consisting with and without exogenous effects in $\sigma$-centrality influence measure

\begin{tabular}{lllll}
\hline$\beta$-levels & \multicolumn{2}{c}{ Without exogenous Effect } & \multicolumn{2}{c}{ With exogenous Effect } \\
\hline & Global Consensus & Optimal $\alpha$-level & Global Consensus & Optimal $\alpha$-level \\
\hline 0 & 0.883 & Level 7 & 0.833 & Level 7 \\
0.1 & 0.895 & Level 7 & 0.899 & Level 7 \\
0.2 & 0.908 & Level 7 & 0.914 & Level 7 \\
0.3 & 0.920 & Level 6 & 0.930 & Level 7 \\
0.4 & 0.933 & Level 7 & 0.939 & Level 7 \\
0.5 & 0.942 & Level 6 & 0.946 & Level 4 \\
0.6 & 0.949 & Level 3 & & \\
\hline
\end{tabular}

The above results show that in the second consensus round the experts form closer relations with each other than in the first consensus round, and consequently a lower number of clusters are obtained with the optimal clustering level decreasing from the original $\alpha_{7}$ to $\alpha_{3}$ and $\alpha_{4}$ for without and with exogenous effect, respectively. These results confirm the advantage of implementing the proposed $\sigma$-centrality influence measure in (i) generating feedback preference values based on the network influencer to enable the group reaching consensus; and (ii)the the nomination of a network influencer does not necessarily rely only on the network connections constructed in SSIN but also on external evaluations from third parties that can be available or desirable to be taken into consideration."

\section{Influence-driven resolution process}

This section describes the two required phases of the influence-driven resolution process associated to the CGDM problem described in the paper: (1) the fusion phase; and (2) the exploitation phase.

\subsection{Fusion phase}

The feedback individual preferences derived from the consensus reaching process are aggregated to obtain the group consensus preference relation. Among the many aggregation operators available, the Ordered Weighted Average (OWA) operator, introduced by Yager [43], has become one of the well-known fusion techniques applied in decision making area. The definition of an OWA operator is presented below:

Definition 5. "An OWA operator of dimension $n$ is a mapping $\phi: \mathbb{R}^{n} \longrightarrow \mathbb{R}$, associated with a weighting vector, $W=\left(\omega_{1}, \ldots, \omega_{n}\right)$, such that $\omega_{i} \in[0,1]$ and $\sum_{i=1}^{n} \omega_{i}=1$, with following expression:

$$
\phi\left(p_{1}, \ldots, p_{n}\right)=\sum_{i=1}^{n} \omega_{i} \cdot p_{\varrho(i)}
$$

where $\varrho:\{1, \ldots, n\} \longrightarrow\{1, \ldots, n\}$ is a permutation function such that $p_{\varrho(i)} \geq p_{\varrho(i+1)}, \forall i=$ $1, \ldots, n-1$, i.e., $p_{\varrho(i)}$ is the $i$-th highest value in the set $\left\{p_{1}, \ldots, p_{n}\right\}$." 
The implementation of the concept of majority is desirable when deriving the final group solution to the CGDM problem. An interesting methodology for doing this was developed by Yager [44] with the quantifier guided linguistic OWA operator. This methodology allows to implement natural language terms such as 'most of', 'some of', 'at least one', 'as many as possible' and 'all' by means of their representation as fuzzy sets on the domain [0,1] with corresponding appropriate increasing monotonic quantifier membership functions $Q:[0,1] \rightarrow$ $[0,1]$ verifying $Q(0)=0, Q(1)=1$ reflecting in general the proportion of criteria/experts satisfied by an acceptable solution [43]. A quantifier guided linguistic OWA operator weights are computed as follows:

$$
w_{i}=Q\left(\frac{i}{n}\right)-Q\left(\frac{i-1}{n}\right), i=1, \ldots, n
$$

Yager [44] further extended the above approach to allow for different importance degrees associated to criteria or experts to be implemented. In this case, the quantifier guided linguistic OWA operator are computed using the following expression:

$$
w_{i}=Q\left(\frac{\sum_{k=1}^{i} \mu_{\varrho}(k)}{Z}\right)-Q\left(\frac{\sum_{k=1}^{i-1} \mu_{\varrho}(k)}{Z}\right)
$$

where $Z=\sum_{k=1}^{n} \mu_{k}$ is the total sum of importance and $\varrho$ is the permutation utilized for the purpose of obtaining the ordering of the values to be fused.

Yager and Filev [29] introduced a more general type of OWA operator, the Induced OWA (IOWA) operator, by inducing the reordering step of the first argument variable upon the magnitude of the second variable, the order inducing variable, which is mathematically defined as below:

Definition 6. "An IOWA operator of dimension $n$ is a function $\Phi_{W}:(\mathbb{R} \times \mathbb{R})^{n} \longrightarrow \mathbb{R}$, to which a weighting vector is associated $W=\left(\omega_{1}, \ldots, \omega_{n}\right)$ such that $\omega_{i} \in[0,1]$ and $\sum_{i} \omega_{i}=1$, with following expression:

$$
\Phi_{W}\left(\left\langle u_{1}, p_{1}\right\rangle, \ldots,\left\langle u_{n}, p_{n}\right\rangle\right)=\sum_{i=1}^{n} \omega_{i} \cdot p_{\varrho(i)}
$$

being $\varrho:\{1, \ldots, n\} \longrightarrow\{1, \ldots, n\}$ a permutation where $p_{\varrho(i)} \geq p_{\varrho(i+1)}, \forall i=1, \ldots, n-1$, i.e., $\left\langle u_{\varrho(i)}, p_{\varrho(i)}\right\rangle$ is the 2-tuple with $u_{\varrho(i)}$ the $i$-th highest value in the set $\left\{u_{1}, \ldots, u_{n}\right\}$."

The influence score of each expert, $Y$, obtained using Definition 3 is proposed in this context as the order inducing variable of the experts' preference evaluations to fuse, $\left\{p_{i j}^{1}, \ldots, p_{i j}^{m}\right\}$, which leads to the following $\sigma$-IOWA operator:

Definition 7. "The $\sigma$-IOWA operator of dimension $m, \Phi_{W}^{\sigma}$, is an IOWA operator with the set of influence score of experts in the network, $Y=\left(y^{1}, \ldots, y^{m}\right)$, as the order inducing variable."

Thus, denoting by $W$ the weighting vector calculated using Equation (18) with $Q$ a fuzzy linguistic quantifier representing the concept of soft majority desired to implement, the collective preference relation derived using the $\sigma-I O W A$ operator, $\Phi_{W}^{\sigma}$, will be

$$
p_{i j}^{c}=\Phi_{W}^{\sigma}\left(\left\langle y^{1}, p_{i j}^{1}\right\rangle, \ldots,\left\langle y^{m}, p_{i j}^{m}\right\rangle\right) .
$$


Cleary, the higher the influence score, the more influence an expert has in the network, and consequently the higher the contribution (weight value) of that expert in the fusion process. Indirectly, the implication of less influential experts can be mitigated. This can be achieved by implementing the concept of fuzzy majority via an increasing concave linguistic quantifier $Q$ [45, 46]. Yager [44] proposed the following parameterized family of RIM quantifiers $Q(r)=$ $r^{a}, a \geq 0$ to model the majority concept 'most of', which is concave when $a \in[0,1]$. For illustrative purpose, the value $a=1 / 2$ will be used, and the collective preferences represent the degree of "preference of one alternative over another for "most of' the influential experts" in the network.

\subsection{Exploitation Phase}

The exploitation phase of the CGDM process aims to rank the alternatives so that the best one can be identified. The OWA Quantifier Guided Dominance Degree (QGDD) [30] is proposed to be applied, which is formally defined as:

Definition 8. "Let $P^{c}=\left(p_{i j}^{c}\right)$ be the collective preference relation of the set of alternatives $\mathbb{A}=\left\{A_{1}, A_{2}, \ldots, A_{n}\right\}$. The quantifier guided dominance degree that alternative $A_{i}$ has over the others, in a fuzzy majority sense, is expressed as below:

$$
Q G D D\left(A_{i}\right)=\Phi_{Q}\left(p_{i j}^{c}, j=1, \ldots, n, j \neq i\right)
$$

being $\Phi_{Q}$ an $O W A$ operator guided by the linguistic quantifier $Q$ representing the fuzzy majority concept."

The set of alternatives with maximum QGDD will be the solution to the influence-based CGDM problem;

$$
\mathbb{A}^{Q G D D}=\left\{A \mid A \in \mathbb{A}, Q G D D(A)=\sup _{A \in \mathbb{A}} Q G D D(A)\right\} .
$$

Algorithm 5 shows the consecutive steps of the proposed influence-driven resolution process.

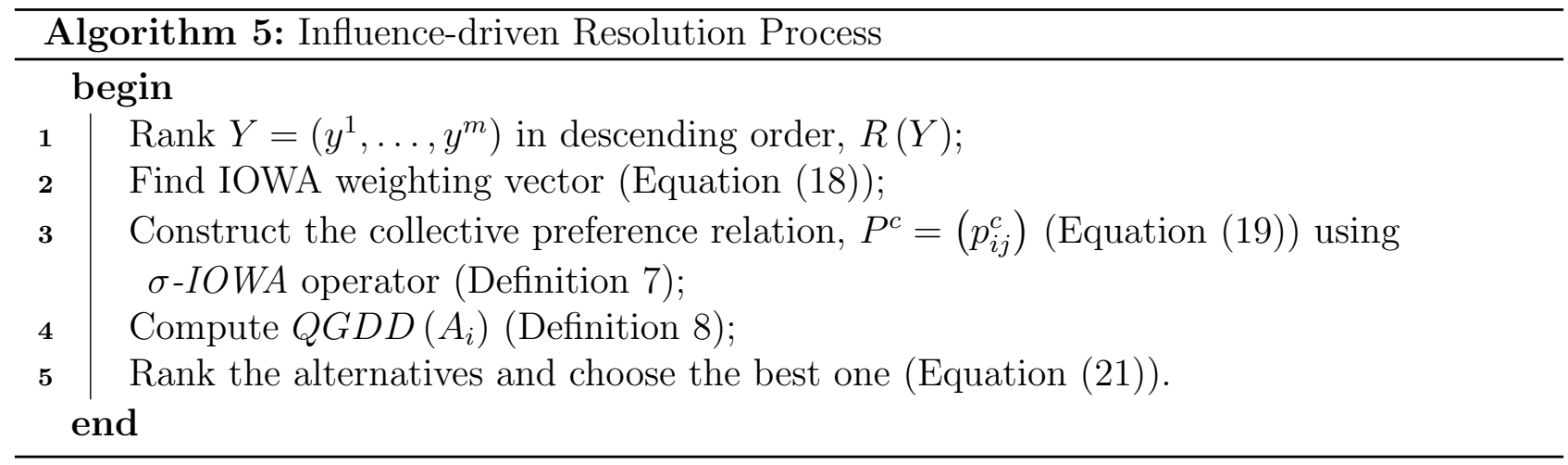

Example 4 (Continuation of Example 3). "Table 4 presents the results obtained after the influence-driven resolution process is implemented without and with exogenous effects. The values obtained for the influence scores (in normalized form), IOWA weighting vectors, collective preferences, maximal dominance degrees and final ranking of alternatives are included. The values obtained without exogenous effect are slightly different to the ones obtained with exogenous effect, which indicated that the exogenous effect absolutely affects the resolution procedure final ranking of alternatives."

Figure 5 provides the general research flow of the proposed influence-driven feedback system for preference similarity network clustering based consensus group decision making model. 
Table 4: Exogenous effects on influence based resolution process

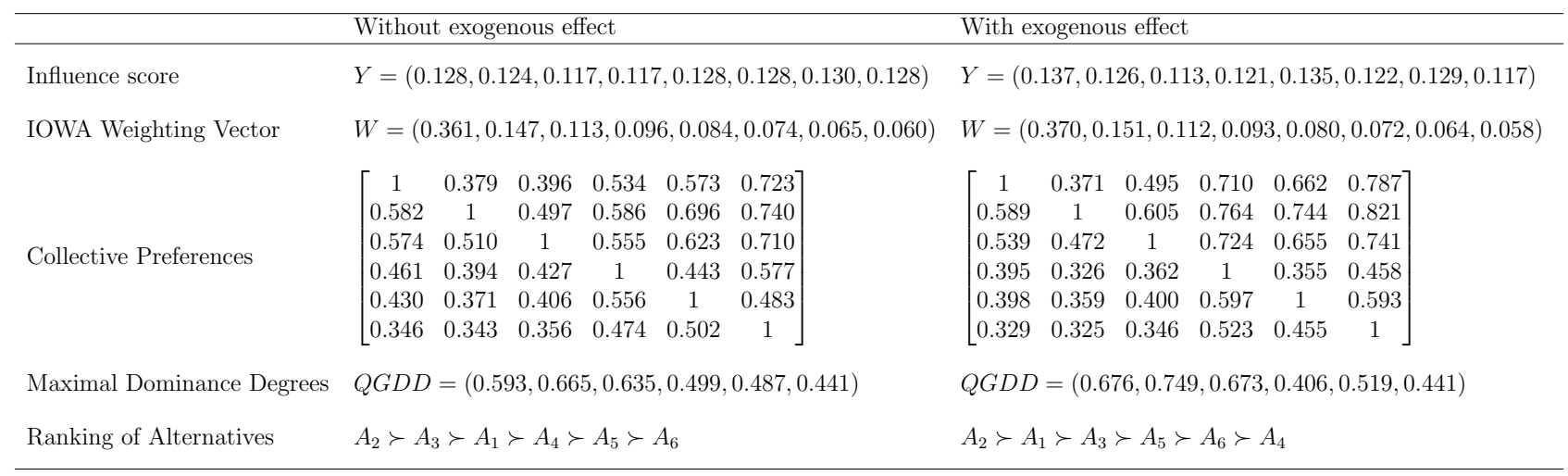

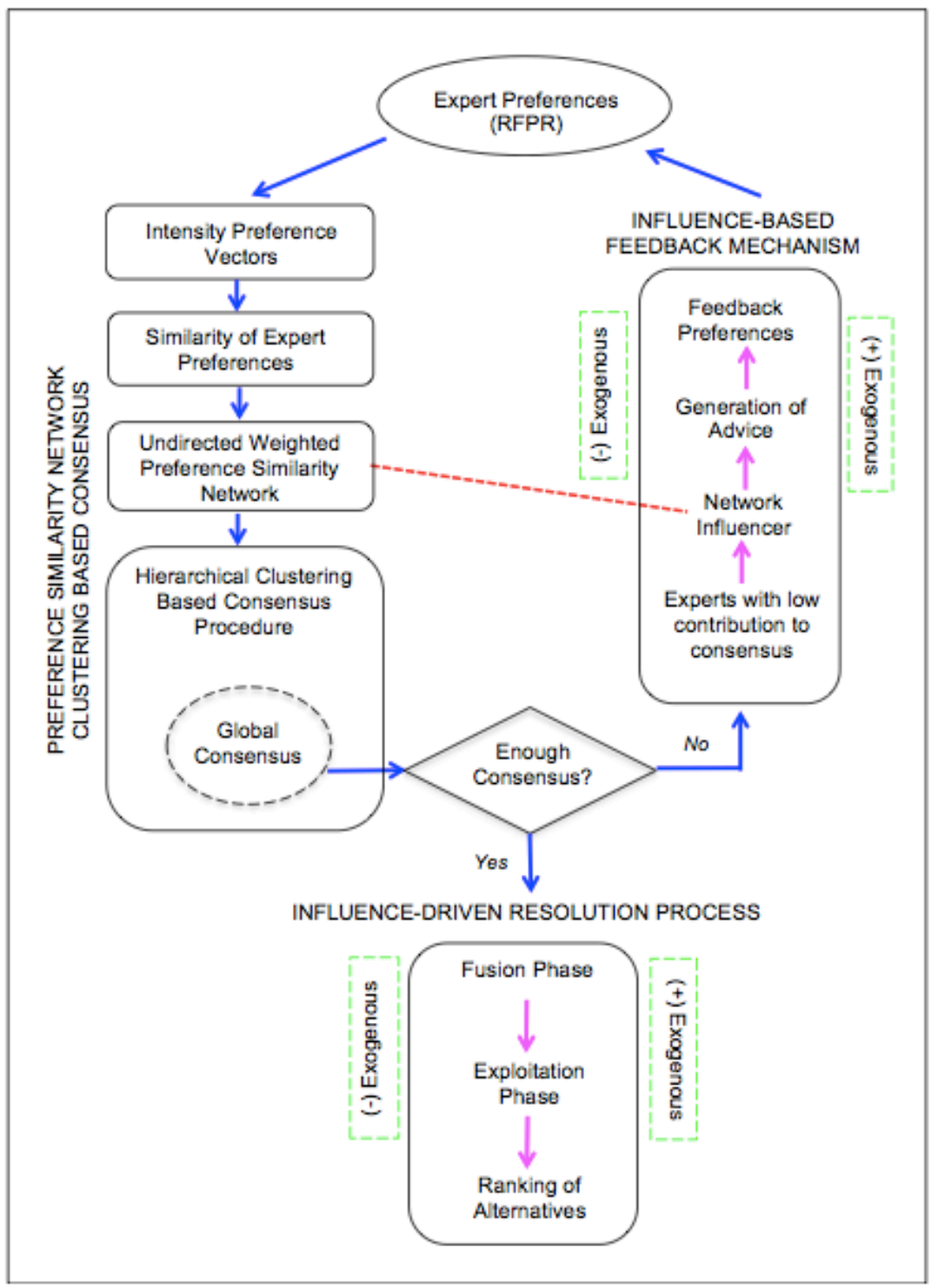

Figure 5: A research flow of the proposed influence-driven feedback system for preference similarity network clustering based consensus group decision making 


\section{Conclusion}

The incorporation of Social Influence Network (SIN) in consensus group decision making (CGDM) models provide new avenues to researchers within this area of study with respect to the introduction of new advanced research decision-making frameworks. We proposed an influence-driven feedback system to be implemented in a preference similarity network clustering based group decision making procedure. Inspired by the introduction of the alpha-centrality eigenvector-like measure by Bonancich and Lloyd [39], we proposed to use this measure in a group decision making context by building an SIN adjacency matrix, based on the preference similarity matrix obtained from the experts' initial evaluations, with the aim of providing feedback and generating advices to experts in a group to support the reaching of consensus. The proposed approach is built on a new influence score measure, called $\sigma$-centrality, and the convergence of the solution is confirmed when there exists a unique highest eigenvalue in the set of eigenvalues from the row normalized preference similarity matrix. This approach ensures in the first place that valuable recommendations are coming from expert with similar preferences. In addition, both endogenous and exogenous factors are comprised in order to express experts' importance weights based on the internal SSIN connections and third parties evaluations, in such a way that a fair nomination of the network influencer is achieved. This influence-driven feedback mechanism positively contributes towards the achievement of sufficient consensus state, with experts with low contribution to consensus being successfully moved closer to each other, following the recommendations that are built from the network influencer's preferences. The feedback process is designed based on the satisfying of two important conditions to ensure that (1) the revised consensus degree is above the consensus threshold and that (2) the clustering solution is improved. Summarizing, the proposed model is an effective CGDM model that considers important elements that are usually overlooked by other existing frameworks.

\section{Acknowledgements}

The authors acknowledge valuable support from the University of Leicester, De Montfort University, Ministry of Higher Education Malaysia and Universiti Teknologi MARA (Malaysia).

\section{References}

[1] M. J. del Moral, Francisco Chiclana, J. M. Tapia, E. Herrera-Viedma, A comparative study on consensus measures in group decision making. International Journal of Intelligent Systems 33 (2018) 1624-1638.

[2] N. Zhang, Z. Gong, F. Chiclana, Minimum cost consensus models based on random opinions, Expert Systems with Applications 89 (2017) 149-159.

[3] Z. Gong, C. Xu, F. Chiclana, X. Xu, Consensus measure with multi-stage fluctuation utility based on china's urban demolition negotiation, Group Decision and Negotiation 26 (2) (2017) 379-407.

[4] T. González-Arteaga, R. de Andrés Calle, F. Chiclana, A new measure of consensus with reciprocal preference relations: The correlation consensus degree, Knowledge-Based Systems 107 (2016) 104-116.

[5] Z. Wu, J. Xu, A consensus model for large-scale group decision making with hesitant fuzzy information and changeable clusters, Information Fusion 41 (2018) 217-231.

[6] Y. Bouzarour-Amokrane, A. Tchangani, F. Peres, A bipolar consensus approach for group decision making problems, Expert Systems with Applications 42 (3) (2015) 1759-1772. 
[7] Z. Gong, N. Zhang, K. W. Li, L. Martínez, W. Zhao, Consensus decision models for preferential voting with abstentions, Computers \& Industrial Engineering 115 (2018) 670682.

[8] X. Tan, Z. Gong, F. Chiclana, N. Zhang, Consensus modeling with cost chance constraint under uncertainty opinions, Applied Soft Computing 67 (2018) 721-727.

[9] Z. Gong, X. Xu, F. Lu, L. Li, C. Xu, On consensus models with utility preferences and limited budget, Applied Soft Computing 35 (2015) 840-849.

[10] J. L. Garcia-Lapresta, D. Perez-Roman, Ordinal proximity measures in the context of unbalanced qualitative scales and some applications to consensus and clustering, Applied Soft Computing 35 (2015) 864-872.

[11] J. L. Garcia-Lapresta, D. Perez-Roman, Consensus-based clustering under hesitant qualitative assessments, Fuzzy Sets and Systems 292 (2016) 261-273.

[12] E. Abel, L. Mikhailov, J. Keane, Clustering decision makers with respect to similarity of views, in: 2014 IEEE Symposium on Computational Intelligence in Multi-Criteria Decision-Making (MCDM), IEEE, 2014, pp. 40-47.

[13] N. H. Kamis, F. Chiclana, J. Levesley, Preference similarity network structural equivalence clustering based consensus group decision making model, Applied Soft Computing 67 (2018) 706-720.

[14] N. H. Kamis, F. Chiclana, J. Levesley, Geo-uninorm consistency control module for preference similarity network hierarchical clustering based consensus model, Knowledge-Based Systems doi: 10.1016/j.knosys.2018.05.039

[15] O. Appel, F. Chiclana, J. Carter, H. Fujita, A consensus approach to the sentiment analysis problem driven by support-based IOWA majority. International Journal of Intelligent Systems 32 (2017) 947-965.

[16] M. Brunelli, M. Fedrizzi, M. Fedrizzi, Fuzzy m-ary adjacency relations in social network analysis: Optimization and consensus evaluation, Information Fusion 17 (2014) 36-45.

[17] Y. Dong, Z. Ding, L. Martínez, F. Herrera, Managing consensus based on leadership in opinion dynamics, Information Sciences 397 (2017) 187-205.

[18] J. Wu, F. Chiclana, H. Fujita, E. Herrera-Viedma, A visual interaction consensus model for social network group decision making with trust propagation, Knowledge-Based Systems 122 (2017) 39-50.

[19] E. Herrera-Viedma, F. J. Cabrerizo, F. Chiclana, J. Wu, M. J. Cobo, S. Konstantin, Consensus in group decision making and social networks, Studies in Informatics and Control 26 (3) (2017) 259-268.

[20] Q. Liang, X. Liao, J. Liu, A social ties-based approach for group decision-making problems with incomplete additive preference relations, Knowledge-Based Systems 119 (2017) 68-86.

[21] N. Capuano, F. Chiclana, H. Fujita, E. Herrera-Viedma, V. Loia, Fuzzy group decision making with incomplete information guided by social influence, IEEE Transactions on Fuzzy Systems 26 (3) (2018) 1704-1718.

[22] L. G. Pérez, F. Mata, F. Chiclana, G. Kou, E. Herrera-Viedma, Modelling influence in group decision making, Soft Computing 20 (4) (2016) 1653-1665. 
[23] J. Wu, F. Chiclana, A social network analysis trust-consensus based approach to group decision-making problems with interval-valued fuzzy reciprocal preference relations, Knowledge-Based Systems 59 (2014) 97-107.

[24] J. Wu, F. Chiclana, E. Herrera-Viedma, Trust based consensus model for social network in an incomplete linguistic information context, Applied Soft Computing 35 (2015) 827-839.

[25] J. Wu, F. Chiclana, Visual information feedback mechanism and attitudinal prioritisation method for group decision making with triangular fuzzy complementary preference relations, Information Sciences 279 (2014) 716-734.

[26] Y. Liu, C. Liang, F. Chiclana, J. Wu, A trust induced recommendation mechanism for reaching consensus in group decision making, Knowledge-Based Systems 119 (2017) 221231.

[27] J. Wu, Y. Liu, C. Liang, A consensus-and harmony-based feedback mechanism for multiple attribute group decision making with correlated intuitionistic fuzzy sets, International Transactions in Operational Research 22 (6) (2015) 1033-1054.

[28] Z. Gong, X. Xu, H. Zhang, U. A. Ozturk, E. Herrera-Viedma, C. Xu, The consensus models with interval preference opinions and their economic interpretation, Omega 55 (2015) 81-90.

[29] R. R. Yager, D. Filev, Operations for granular computing: mixing words and numbers, in: Proceedings of the 1998 IEEE World Congress on Computational Intelligence, Vol. 1, IEEE, 1998, pp. 123-128.

[30] F. Chiclana, F. Herrera, E. Herrera-Viedma, Integrating three representation models in fuzzy multipurpose decision making based on fuzzy preference relations, Fuzzy Sets and Systems 97 (1) (1998) 33-48.

[31] N. Capuano, F. Chiclana, E. Herrera-Viedma, H. Fujita, V. Loia, Fuzzy rankings for preferences modeling in group decision making. International Journal of Intelligent Systems 33 (2018) 1555-1570.

[32] S. P. Borgatti, T. J. Grosser, Structural equivalence: Meaning and measures, in: International Encyclopedia of the Social \& Behavioral Sciences, 2nd Edition, Elsevier, 2015, pp. 621-625.

[33] J. R. French Jr, A formal theory of social power, Psychological review 63 (3) (1956) 181.

[34] F. Harary, A criterion for unanimity in french's theory of social power., in: D. Cartwright (Ed.), Studies in social power, Univer. Michigan, Oxford, England, 1959, pp. 168-182.

[35] M. H. DeGroot, Reaching a consensus, Journal of the American Statistical Association 69 (345) (1974) 118-121.

[36] N. E. Friedkin, E. C. Johnsen, Social influence and opinions, Journal of Mathematical Sociology 15 (3-4) (1990) 193-206.

[37] Y. Dong, Q. Zha, H. Zhang, G. Kou, H. Fujita, F. Chiclana, E. Herrera-Viedma, Consensus reaching in social network group decision making: Research paradigms and challenges, Knowledge-Based Systems doi: 10.1016/j.knosys.2018.06.036

[38] N. E. Friedkin, E. C. Johnsen, Social influence networks and opinion change, Advances in group processes 16 (1) (1999) 1-29. 
[39] P. Bonacich, P. Lloyd, Eigenvector-like measures of centrality for asymmetric relations, Social networks 23 (3) (2001) 191-201.

[40] J. Wu, L. Dai, F. Chiclana, H. Fujita, E. Herrera-Viedma, A minimum adjustment cost feedback mechanism based consensus model for group decision making under social network with distributed linguistic trust, Information Fusion 41 (2018) 232-242.

[41] X. Su, T. M. Khoshgoftaar, A survey of collaborative filtering techniques, Advances in artificial intelligence 2009.

[42] B. K. Tareen, J.-w. Lee, S.-g. Lee, Synergy: a workbench for collaborative filtering algorithms on user interaction data, in: International Workshop on User Data Interoperability in the Social Web, 2010, p. 9 pages.

[43] R. R. Yager, On ordered weighted averaging aggregation operators in multicriteria decisionmaking, IEEE Transactions on Systems, Man, and Cybernetics 18 (1) (1988) 183-190.

[44] R. R. Yager, Quantifier guided aggregation using owa operators, International Journal of Intelligent Systems 11 (1) (1996) 49-73.

[45] F. Chiclana, E. Herrera-Viedma, F. Herrera, S. Alonso, Some induced ordered weighted averaging operators and their use for solving group decision-making problems based on fuzzy preference relations, European Journal of Operational Research 182 (1) (2007) 383399.

[46] F. Chiclana, F. Mata, L. G. Perez, E. Herrera-Viedma, Type-1 OWA unbalanced fuzzy linguistic aggregation methodology. application to eurobonds credit risk evaluation. International Journal of Intelligent Systems 33 (2018) 1071-1088. 